\title{
Selective area growth of $a$ - and $c$-plane GaN nanocolumns by molecular beam epitaxy using colloidal nanolithography
}

\author{
A. Bengoechea-Encabo ${ }^{a, *}$, S. Albert ${ }^{a}$, M.A. Sanchez-Garcia ${ }^{a}$, L.L. López ${ }^{b}$, S. Estradé ${ }^{b, c}$, J.M. Rebled $^{\text {b,d }}$, \\ F. Peiró ${ }^{b}$, G. Nataf ${ }^{e}$, P. de Mierry ${ }^{e}$, J. Zuniga-Perez ${ }^{\mathrm{e}}$, E. Calleja ${ }^{\mathrm{a}}$ \\ a ISOM and Departamento de Ingenieria Electronica, ETSIT, Universidad Politécnica, 28040 Madrid, Spain \\ ${ }^{\mathrm{b}}$ LENS, MIND-IN2UB, Departament d'Electrònica, Universitat de Barcelona, Martí i Franquès 1, 08028 Barcelona, Spain \\ c MAT, CCiT, Universitat de Barcelona, Solé i Sabarís 1, 08028 Barcelona, Spain \\ ${ }^{\mathrm{d}}$ Institut de Ciència de Materials de Barcelona-CSIC, Campus UAB, 08193 Bellaterra, Spain \\ e CRHEA-CNRS, Rue Bernard Gregory, 06560 Valbonne, France
}

Keywords:

A1. Nanostructures

A3. Molecular beam epitaxy

A3. Selective epitaxy

B1. Nitrides

\begin{abstract}
A B S T R A C T
Selective area growth of $a$-plane GaN nanocolumns by molecular beam epitaxy was performed for the first time on a-plane GaN templates. Ti masks with $150 \mathrm{~nm}$ diameter nanoholes were fabricated by colloidal lithography, an easy, fast and cheap process capable to handle large areas. Even though colloidal lithography does not provide a perfect geometrical arrangement like e-beam lithography, it produces a very homogeneous mask in terms of nanohole diameter and density, and is used here for the first time for the selective area growth of GaN. Selective area growth of $a$-plane GaN nanocolumns is compared, in terms of anisotropic lateral and vertical growth rates, with GaN nanocolumns grown selectively on the $c$-plane.
\end{abstract}

The main advantages of one-dimensional structures such as nanocolumns, compared to thin films, is the dislocation- and strain-free growth on different substrates. This higher crystal quality yields a better efficiency in devices such as light emitting diodes (LEDs) based on InGaN/GaN quantum disks (QDisks) [1-4].

Much effort has been dedicated to the growth of self-assembled nanocolumns (NCs) by Plasma-Assisted Molecular Beam Epitaxy (PAMBE), allowing a better knowledge of the material physical properties and growth mechanisms [5-8]. However, the strong morphology dispersion, typical of a self-assembled process, hinders both the processing of nanodevices arrays and their electrical behavior (NCs merging, defect generation, current injection inhomogeneities). Indeed, arrays of self-assembled nanoLEDs show in general much lower electroluminescence efficiency than the corresponding one measured by photoluminescence (PL). In addition to that InGaN active regions embedded in self-assembled NCs of different diameters and lengths always show fluctuations in the composition, leading to multicolor emission. Arrays of nanostructures grown by selective area growth (SAG) provide a much better homogeneity in terms of morphology, electrical and optical characteristics. SAG of
III-nitride NCs [9-12] and other nanostructures [13,14], is generally performed on very thin metal (Ti, Mo) or dielectric $\left(\mathrm{SiO}_{2}, \mathrm{SiN}\right)$ masks with an array of nanoholes.

A relevant issue concerning optoelectronic devices based on III-nitrides is the presence of strong polarization fields that may reduce efficiency. This is the case in layers grown along the $c$-axis and, a huge effort is nowadays dedicated to the growth of high quality non-polar and semi-polar material [15], with a particular emphasis on non-polar LEDs [16].

In the case of SAG of GaN NCs on c-plane GaN templates, the growth front is generally formed by semi-polar ( $r$-planes) that yield a "pencil-like" profile [12]. This profile is then transferred to InGaN QDisks embedded within the GaN NC. Though in this structure the effects of internal fields in the active region of the device (i.e. nanoLED) may be reduced compared with polar planes, the most effective solution is to grow along non-polar directions, either by growing core-shell heterostructures on the lateral nonpolar facets of otherwise polar NCs, or by growing axial heterostructures along nonpolar directions such as [10-10] ( $m$-planes) or [11-20] (a-planes). In both approaches, the internal fields are nominally zero.

This work reports on the SAG of a-plane GaN NCs (i.e. the growth direction is parallel to [11-20]) by PAMBE on Ti masks. The vertical and lateral growth rates (anisotropy) are studied and compared with c-plane GaN NCs SAG. 
Nanohole masks are fabricated by colloidal lithography [17-19] on $a$-plane and c-plane GaN templates. Colloidal solution is formed by polyestirene (PS) nanobeads (260 $\mathrm{nm}$ of average diameter and sulfate covered, so negatively charged, and purchased at Invitrogen). Commercial solution is then diluted in water at $4 \% \mathrm{w} / \mathrm{v}$. On the other side, GaN templates were alternately dipped in poly(sodium 4-styrenesolfonate) (PSS) and poly(diallyldimethylammonium chloride) (PDDA) solutions ( $2 \% \mathrm{w} / \mathrm{v}$, during $1 \mathrm{~min}$ each step), which are negatively and positively charged poly-electrolytes, respectively. Poly-electrolytes are purchased at Sigma-Aldrich. A tri-layer PSS/ PDDA/PSS ( $\sim 5 \mathrm{~nm}$ thick) is formed to achieve a negatively charged (like the nanobeads surface) and hydrophilic surface. The GaN/PSS/ PDDA/PSS surface is covered with the colloidal solution by spincoating $(2000 \mathrm{rpm}$ for $30 \mathrm{~s})$. The result is a monolayer of PS nanobeads, with a close-packaged arrangement in large areas (domains up to $400 \times 400 \mu \mathrm{m}^{2}$ ). Once the nanobeads are arranged on the surface, reactive ion etching (RIE) oxygen plasma ( $40 \mathrm{~W}$; oxygen pressure of $80 \mathrm{mTorr}, 1 \mathrm{~nm} / \mathrm{s}$ ) is used in order to reduce the diameter of the beads and, consequently, to isolate them. After the plasma etching, $7 \mathrm{~nm}$ of Ti is evaporated on the surface and a lift-off process results in an array of $150 \mathrm{~nm}$ diameter nanoholes on the Ti layer covering the GaN surface. The colloidal lithography process is depicted in Fig. 1.

During the PAMBE growth (Riber Compaq 21) the substrate temperature was monitored with a thermocouple located at the growth stage. The $\mathrm{Ga}$ and $\mathrm{N}$ fluxes were calibrated in equivalent (0001) GaN growth rate units for compact layers $(\mathrm{nm} / \mathrm{min})$ [7,12,20,21]. Scanning electron microscopy (SEM) images were obtained with a CRESTEC CABL-9500C system. Atomic force microscopy (AFM) images were taken with a Nanoscope microscope (MMAFM-2) and processed with Nanotec software WS $\times$ M [22]. Based on these measurements the growth rates were estimated. Also transmission electron microscopy (TEM) measurements in high resolution (HREM) and selected area electron diffraction modes (SAED) were carried out in a JEOL2010F and a Jeol2100 instruments respectively, in order to confirm the crystal orientation.

Previously to the GaN SAG, a nitridation step was performed following the process reported in Ref. $[10,12]$ to prevent Ti mask degradation at high temperature. Growth time was either $1 \mathrm{~h}$ or $3 \mathrm{~h}$ at $880^{\circ} \mathrm{C}$ with $\Phi_{\mathrm{Ga}}=16 \mathrm{~nm} / \mathrm{min}$ and $\Phi_{\mathrm{N}}=5 \mathrm{~nm} / \mathrm{min}$ or $10 \mathrm{~nm} / \mathrm{min}$. Nominal flux ratios $\left(\Phi_{\mathrm{G}} / \Phi_{\mathrm{N}}\right)$ were always larger than one, but due to the high growth temperatures used for SAG the actual $\Phi_{\mathrm{G}} / \Phi_{\mathrm{N}}$ ratio at nanoholes (growth front) was significantly reduced [12].

Fig. 2 shows SEM images of SAG GaN NCs along the $c$-plane (Fig. 2(a and b)) and $a$-plane (Fig. 2(c and d)), grown under the same conditions: $1 \mathrm{~h}$ at $880^{\circ} \mathrm{C}, \Phi_{\mathrm{Ga}}=16 \mathrm{~nm} / \mathrm{min}$ and $\Phi_{\mathrm{N}}=5 \mathrm{~nm} / \mathrm{min}$. In both cases, perfect selectivity was achieved, but morphology differences were clearly observed. c-Plane GaN NCs had a typical hexagonal structure with pyramidal top surfaces (pencil-like), as expected from previous results [9-14]. However, nanostructures grown on $a$-plane substrates did show elongated geometries with several lateral facets, as observed by SEM and AFM (inset in Fig. 2(c)). The vertical growth rates measured for $c$ - and $a$-plane SAG were of $3.5 \mathrm{~nm} / \mathrm{min}$ and $1.3 \mathrm{~nm} / \mathrm{min}$, respectively. In fact, the measured ratio between both growth rates (2.7) is in the range of the factor derived from the kinetic Wulffs plots ( $v$-plots) calculated for GaN in Ref. [23] (factor 4).

In order to check the morphology evolution of the $a$-plane nanostructures with time and growth conditions, two new samples were grown: (a) like that on Fig. 2(c and d) $\left(880^{\circ} \mathrm{C}\right.$ $\Phi_{\mathrm{Ga}}=16 \mathrm{~nm} / \mathrm{min}$ and $\Phi_{\mathrm{N}}=5 \mathrm{~nm} / \mathrm{min}$ ) but during $3 \mathrm{~h}$, and (b) during $1 \mathrm{~h}$ but under higher $\Phi_{\mathrm{N}}(10 \mathrm{~nm} / \mathrm{min})$. Both samples did show similar morphologies (Fig. 3(a and b)) evolving from elongated (Fig. 2(c)) to arrow-headed shape, similar to those reported by MOVPE [23]. The main difference is that the sample with higher $\Phi_{\mathrm{N}}$ showed the onset of the nanostructures coalescence. Fig. 3(c-e) shows magnified images with the evolution sequence. It is worth to mention the strong lateral growth anisotropy observed in the a-plane nanostructures. Regarding the height of the nanostructures in both cases, either increasing the active nitrogen by a factor of two, or the growth time by a factor of three, led to a proportional increase of height.

A thin foil TEM lamella has been prepared using focused ion beam (FIB). The cross-section was obtained across the direction perpendicular to that of the elongation of the nanocolumns (it is orthogonal to the arrow in Fig. 3(e)). Fig. 4(a) presents a general

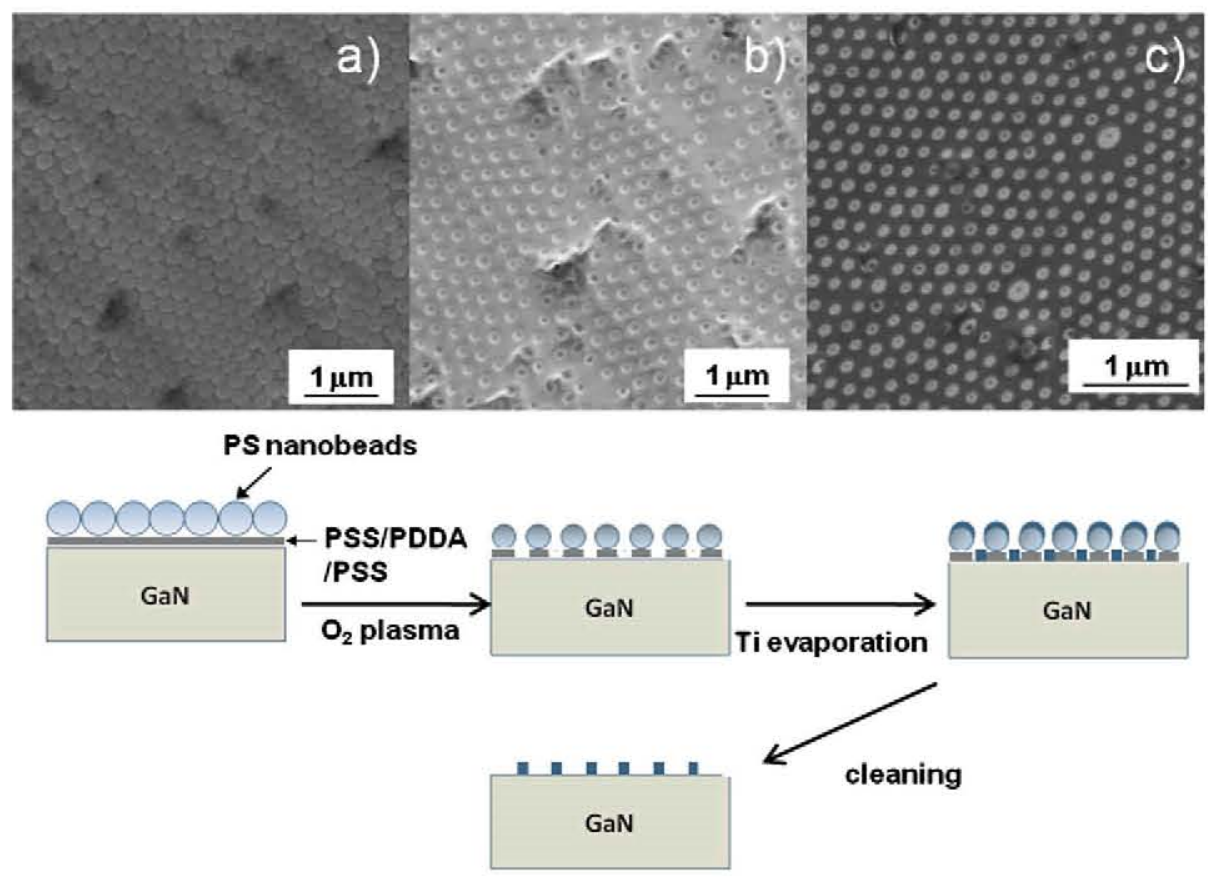

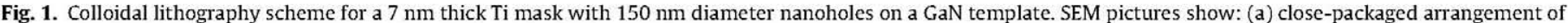
PS nanobeads; (b) after oxygen plasma etching and (c) the final Ti mask. 

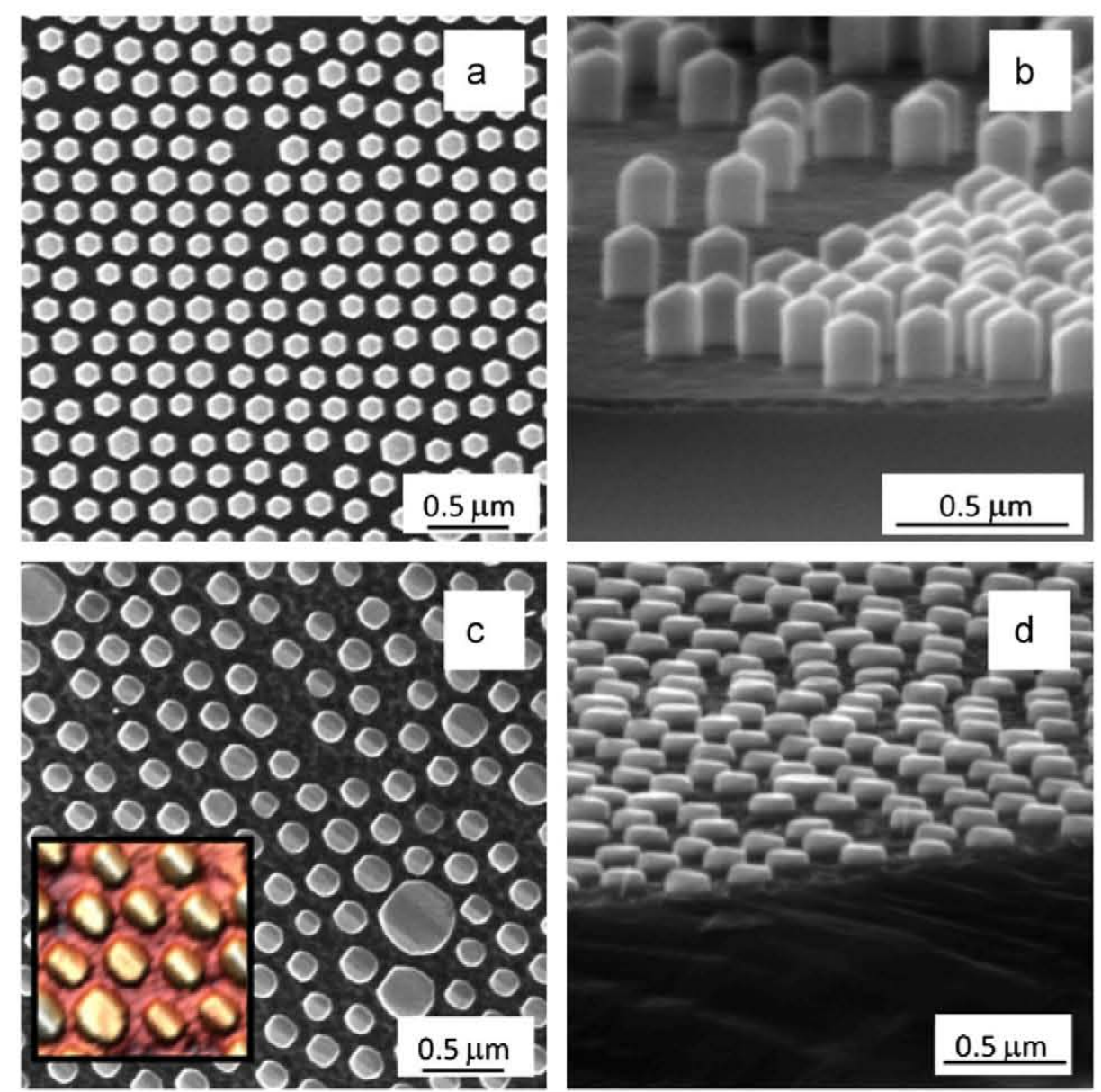

Fig. 2. Plane view and cross section SEM pictures of: ( $a$ and b) c-plane SAG GaN NCs and (c and d) $a$-plane SAG GaN NCs. The inset in Fig. 2 (c) corresponds to an AFM measurement.
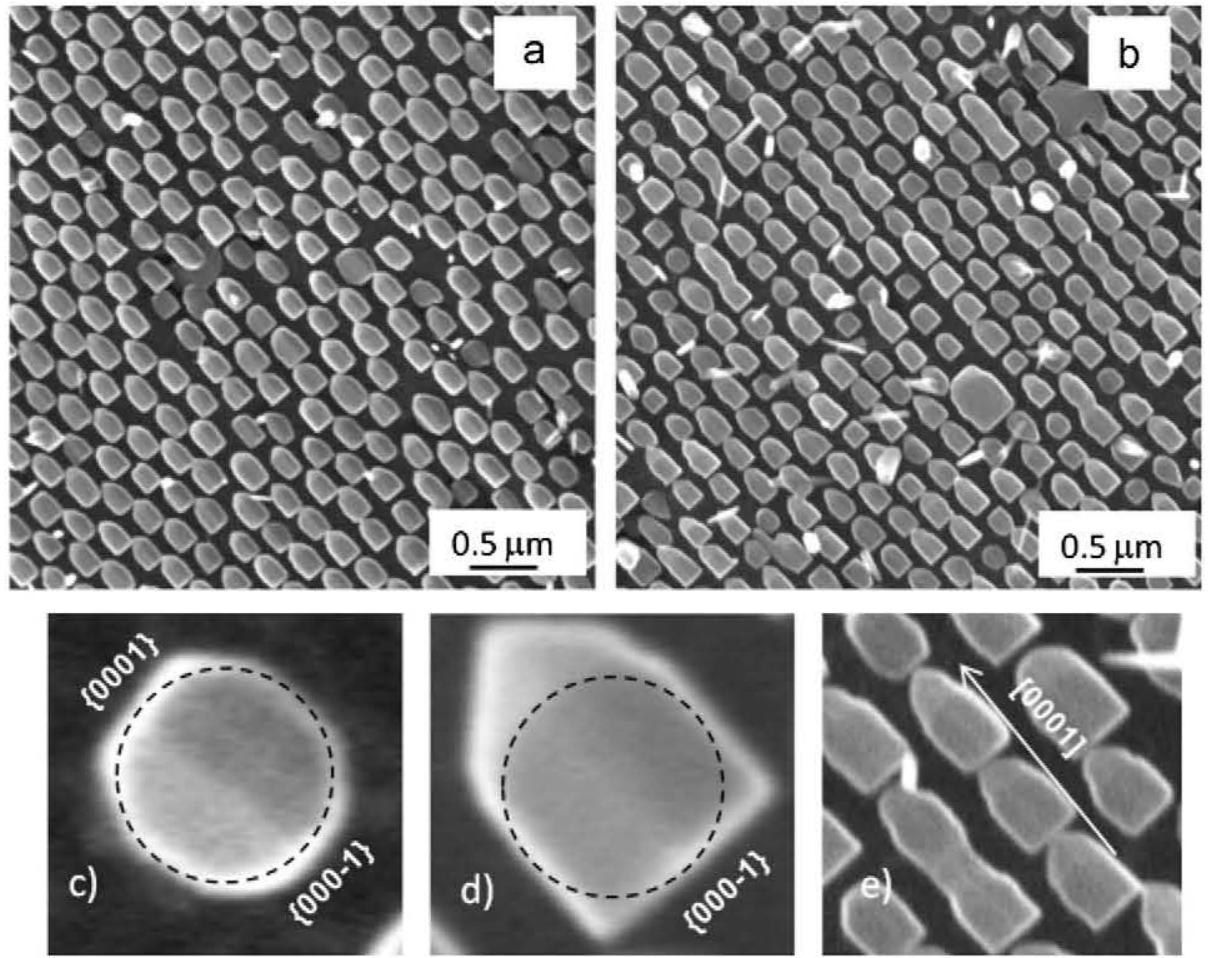

Fig. 3. SEM pictures of $a$-plane SAG GaN NCs grown under the same conditions as in Fig. 2(c) but: (a) twice as much active nitrogen and (b) same active nitrogen but after $3 \mathrm{~h}$ growth. Magnified images of the typical morphology are shown in: (c) for the growth of Fig. 2(c); (d) for the growth in Fig. 3(a) and (e) for the growth in Fig. 3(b). 

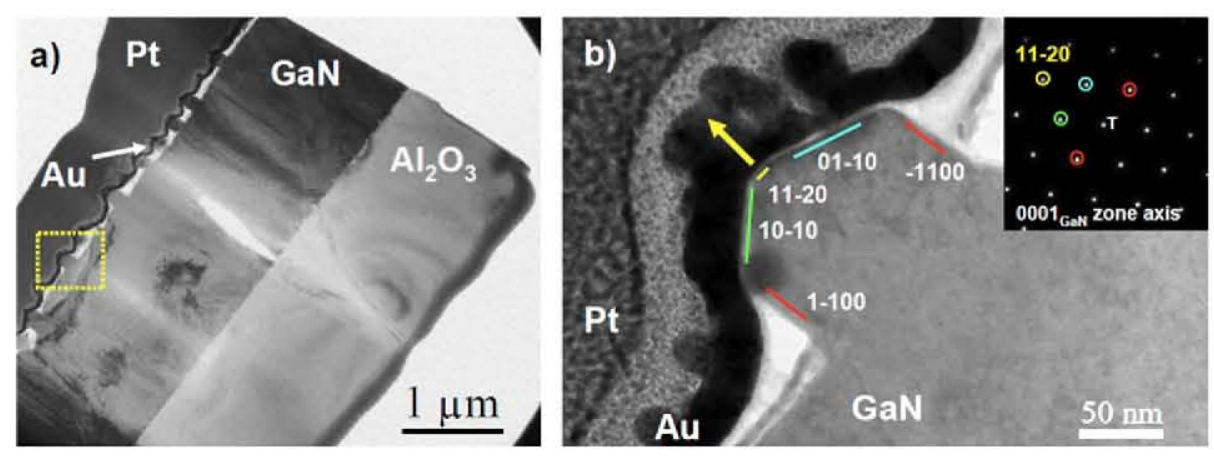

Fig. 4. (a) General view of the lamella prepared using focused ion beam in cross-section perpendicular to the arrow of Fig. 3(e); (b) view of the nanocolumn squared in (a) exhibiting faceting along planes perpendicular to corresponding g vectors for most of the spots in 0001 axis diffraction pattern (inset).

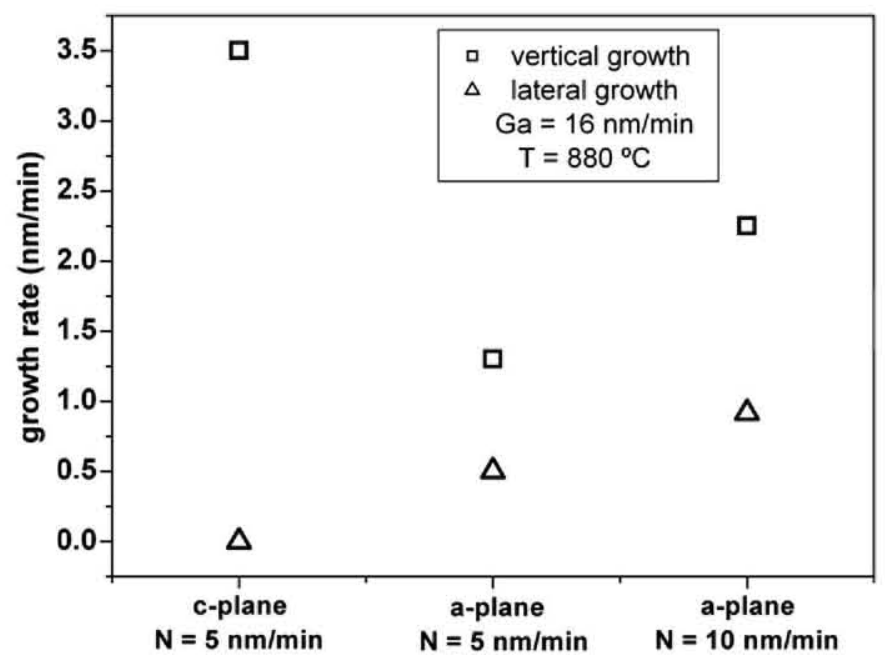

Fig. 5. Vertical and lateral growth rates for GaN NCS SAG on c-plane (growth temperature $880^{\circ} \mathrm{C}, \Phi_{\mathrm{Ga}}=16 \mathrm{~nm} / \mathrm{min}, \Phi_{\mathrm{N}}=5 \mathrm{~nm} / \mathrm{min}$ ), $a$-plane under the same growth conditions and $a$-plane under double active nitrogen amount $(10 \mathrm{~nm} / \mathrm{min})$.

view of the whole lamella where Pt and Au layers, previously deposited to protect the sample surface from ion beam during the thinning process, are clearly observed. In Fig. 4(b), an isolated nanocolumn is presented at higher magnification. The growth direction is confirmed to be [11-20], perpendicular to the a-plane. The nanocolumn exhibits lateral faceting perpendicular to [1-100] and top facets perpendicular to [10-10] and [01-10], i.e. $m$-planes. The corresponding selected area diffraction pattern along the [0001] axis is shown in the inset, confirming the elongation of the nanocolumns in that direction.

Fig. 5 shows the measured vertical and lateral growth rates for $c$-plane GaN and $a$-plane GaN nanostructures (in the last case, lateral growth was measured along the arrow direction, [0001], that corresponded to the largest value) under the two different active nitrogen conditions. While the lateral growth for $c$-plane NCs was almost negligible, as expected [12], in the case of $a$-plane nanostructures the lateral growth rate was almost half the vertical one (lateral to vertical ratio, approximately 0.4 ). A similar ratio was observed in $a$-plane nanostructures when the active nitrogen is doubled.

To summarize, we have demonstrated for the first time the successful use of colloidal Ti masks for SAG of GaN nanostructures by PAMBE. As far as we know, we report for the first time the PAMBE SAG of $a$-plane GaN nanostructures, which is compared with $c$-plane GaN SAG NCs. Consistent with results obtained by other epitaxial techniques, vertical growth rate is slower when growing on a-plane as compared to $c$-plane, under the same growth conditions. In addition, lateral growth is negligible for SAG growth on c-plane, but it turns into significant values in the case of $a$-plane. These achievements may represent the first steps for future optoelectronic applications, where, in addition to an optimal crystal quality, internal electric fields can be avoided.

\section{Acknowledgments}

We acknowledge partial funding by national projects CAMP2009/ ESP-1503, MICINN-PLE2009-0023, MICINN-MAT-2008-04815 and MAT2010-16407, MICINN Consolider CSD2006-19 and CSD200900013 and by UE CP-IP 228999-2 (SMASH).

\section{References}

[1] A. Kikuchi, M. Kawai, M. Tada, K. Kishino, Japanese Journal of Applied Physics 43 (12A) (2004) L1524.

[2] H. Sekiguchi, K. Kato, J. Tanaka, A. Kikuchi, K. Kishino, Physica Status Solidi (a) 205 (2008) 1067.

[3] H.-W. Lin, Yu-Jung Lu, H.-Y. Chen, H.-M. Lee, S. Gwo, Applied Physics Letters 97 (2010) 073101.

[4] W. Guo, M. Zhang, A. Banerjee, P. Bhattacharya, Nano Letters 10 (2010) 3355.

[5] M. Yoshizawa, A. Kikuchi, M. Mori, N. Fujita, K. Kishino, Japanese Journal of Applied Physics 36 (1997) L459.

[6] M.A. Sanchez-García, E. Calleja, E. Monroy, F.J. Sanchez, F. Calle, E. Muñoz, R. Beresford, Journal of Crystal Growth 183 (1998) 23.

[7] S. Fernández-Garrido, J. Grandal, E. Calleja, M.A. Sánchez-García, D. LópezRomero, Journal of Applied Physics 106 (2009) 126102.

[8] J. Ristic, E. Calleja, S. Fernandez-Garrido, L. Cerutti, A. Trampert, U. Jahn, K.H. Ploog, Journal of Crystal Growth 310 (18) (2008) 4035.

[9] H. Sekiguchi, K. Kishino, A. Kikuchi, Applied Physics Express 1 (2008) 124002.

[10] K. Kishino, H. Sekiguchi, A. Kikuchi, Journal of Crystal Growth 311 (2009) 2063

[11] H. Sekiguchi, K. Kishino, A. Kikuchi, Applied Physics Letters 96 (2010) 231104.

[12] A. Bengoechea-Encabo, F. Barbagini, S. Fernandez-Garrido, J. Grandal, J. Ristic, M.A. Sanchez-Garcia, E. Calleja, U. Jahn, E. Luna, A. Trampert, Journal of Crystal Growth 325 (2011) 89.

[13] K. Tachibana, T. Someya, S. Ishida, Y. Arakawa, Applied Physics Letters. 76 (22) (2000) 3212.

[14] T. Kim, J. Kim, M.-S. Yang, S. Lee, Y. Park, U-In Chung, Y. Cho, Applied Physics Letters 97 (2010) 241111.

[15] P. de Mierry, N. Kriouche, M. Nemoz, S. Chenot, G. Nataf, Applied Physics Letters 96 (2010) 231918.

[16] Kwang-Choong Kim, Mathew C. Schmidt, Hitoshi Sato, Feng Wu, Natalie Fellows, Zhongyuan Jia, Makoto Saito, Shuji Nakamura, Steven P. DenBaars, James S. Speck, Kenji Fujito, Applied Physics Letters 91 (2007) 181120.

[17] H.W. Deckman, J.H. Dunsmuuir, Applied Physics Letters 41 (1982) 3.

[18] J.C. Hulteen, R.P. Vanduyne, Journal of Vacuum Science \& Technolology A: Vacuum, Surfaces, and Films 13 (1995) 1553.

[19] H. Li, J. Low, K.S. Brown, N. Wu, IEEE Sensors Journal 8 (6) (2008) 880.

[20] B. Heying, R. Averbeck, L.F. Chen, E. Haus, H. Riechert, J.S. Speck, Journal of Applied Physics 88 (2000) 1855.

[21] G. Koblmüller, S. Fernández-Garrido, E. Calleja, J.S. Speck, Applied Physics Letters 91 (2007) 161904

[22] I. Horcas, R. Fernandez, J.M. Gomez-Rodriguez, J. Colchero, J. Gomez-Herrero A.M. Baro, Review of Scientific Instruments 78, 013705 (2007). Software Nanotec at $\langle$ http://www.nanotec.es/ $\rangle$.

[23] V. Jindal, F. Shahedipour-Sandvik, Journal of Applied Physics 106 (2009) 083115. 